to the photobleaching measurements, some 25 per cent of the bungarotoxinacetylcholine receptor complexes were immobile on the time scale of the measurement, another finding not confirmed by Poo's new experiments. These discordant results raise the possibility that the macromolecular ligand, $\alpha$-bungarotoxin, has retarded the lateral mobility of the native receptor either by direct interaction with other cell surface components or, indirectly, by enhancing the ability of the receptor to interact with other cellular components. Alternatively, the rate of diffusion might be reduced by a photochemical process unique to this labelled ligand-receptor combination in the muscle cell membrane.

While photobleaching techniques have so far been largely directed towards problems in membrane biology, an exciting development has been their application to the study of macromolecules in solution and within the cytoplasm of living cells. At Syracuse University, photobleaching techniques are being used to study the polymerization of actin monomers in solution by monitoring the growth of the immobile fraction with time and should

\section{Gravitational lenses}

\section{from Charles Alcock}

ONE of the most exciting recent developments in astronomy has been the discovery of multiple imaging of quasars. There are three confirmed examples ${ }^{1-3}$ of this phenomenon, and in one case, the gravitational lens responsible for the multiple imaging has been detected ${ }^{4}$. It is a large elliptical galaxy near the centre of a cluster of galaxies.

The great virtue of the phenomenon is that, by the standards of extragalactic astronomy, it can be fairly unambiguously interpreted. In each case the optical emission line spectra of the different images are essentially the same and have identical redshifts.

The general properties of gravitational lenses are easy to understand. A galaxy or cluster of galaxies will bend light rays through small angles, producing (in general) an odd number of images of a background quasar. Usually there will be only one image, but fortuitous projection of a quasar behind a galaxy can result in three or more images. The image brightnesses differ from the brightnesses that would be observed if the lens was absent because the beams are focused.

Three papers in a recent issue of the Astrophysical Journal explore some more

Charles Alcock is Associate Professor in the Department of Physics, 6-207, Massachusetts Institute of Technology, Cambridge, Massachusetts 02139. provide a useful alternative to conventional viscometric techniques. Further, the association of fluorescently derivitized actin with existing fibres in live cells is being examined at the Weizmann Institute using a combination of microinjection and photobleaching techniques. In another novel application, investigators at the University of North Carolina and Penn State University have shown that the diffusion of macromolecules, such as IgG and bovine serum albumin, microinjected into the cytoplasm of living fibroblasts is retarded 70-fold compared with aqueous buffer values; these results suggest that the cytoplasmic matrix hinders the free diffusion of such molecules sterically and/or through binding interactions between the diffusant and the matrix.

These emerging applications of the photobleaching techniques to the study of molecular movement both within cell membranes and within the cytoplasm suggest a great potential for this approach to provide unique dynamic information on cellular structures, particularly when these measurements can be interpreted in the context of available biochemical and ultrastructural data.

speculative suggestions involving gravitational lenses. In the first, Tyson ${ }^{5}$ revives an old suggestion of Barnothy and Barnothy that quasars may be the nuclei of Seyfert galaxies and appear very luminous because an intervening gravitational lens has amplified the flux from the nucleus. The apparent increase in the density of quasars with increasing redshift can then be accounted for by the larger probability that amplification will occur if the Seyfert galaxy is at a greater distance. What is new in Tyson's approach is that he uses his own data (obtained using automated pattern recognition techniques) on the number density of galaxies on the surface of the celestial sphere as a function of measured flux to calculate the logarithmic slope of the number density of quasars as a function of measured flux. The answer, d log $N_{\mathrm{Q}} / \mathrm{d} m_{\mathrm{Q}}=0.9$ (where $m_{\mathrm{Q}}=2.5 \log$ flux) is within the range of observed slopes (0.86-0.95).

The primary difficulty with this model is that the large ( $>15$ times) amplification required is difficult to obtain with gravitational lenses. Furthermore, high amplification should be accompanied by multiple imaging but multiple images have only been detected in three cases out of the nearly 1,000 known quasars. In addition there would need to be a large number of distant galaxies, at present undetected, capable of producing amplification to explain the observed surface density of quasars. Since only a minority of the galaxies we see locally appear capable of producing large amplifications, the mean density of the Universe has to be very high if the model is to work. Success in predicting $\mathrm{d} \log N_{\mathrm{Q}} / \mathrm{d} m_{\mathrm{Q}}$ is not sufficient to remove these doubts.

In the second of the papers Avni ${ }^{6}$ addresses a closely related point. $\mathrm{He}$ discusses Turner's suggestion that amplification by gravitational lenses may significantly perturb the measured statistics of quasars, perhaps accounting for the apparent increase in the number density of quasars with increasing redshift. Quasar analyses are customarily performed on flux-limited samples, which will preferentially include quasars whose flux has been amplified by an intervening gravitational lens. Avni's improvement on Turner's model is to require average flux conservation, which implies that the mean amplification has to be unity. This is an important, obvious requirement which considerably reduces the effect of lenses on the quasar statistics below that computed by Turner. Avni correctly concludes that it is unlikely that the gravitational lens effect can account for the apparent evolution of the density of quasar with redshift.

The third paper takes a different direction. Paczyński and Górski ${ }^{7}$ suggest that a cluster of quasars ${ }^{8}$ at redshift 2.05 may be another example of multiple imaging by a gravitational lens. However, there are some difficulties - the objects have a large separation (minutes, rather than seconds, of arc) and their redshifts are different (by about $10^{3} \mathrm{~km} \mathrm{~s}^{-1}$ ). The authors produce model lenses consisting of two large clusters of galaxies which can account for the large angular separations. The model cannot account for the redshift differences but the time delays between the three images are $10^{2}-10^{3}$ years, and it is suggested that the quasar's redshift might change during this time interval.

The velocity dispersions of the two clusters of galaxies in the models of Paczyński and Górski are large $(>1,500 \mathrm{~km}$ $\left.\mathrm{s}^{-1}\right)$. They point out that if there is a positive cosmological constant the necessary velocity dispersion decreases. This suggests that, should the clusters be discovered and their velocity dispersion measured, one could then estimate the cosmological constant.

It is clear that gravitational lenses can in principle do many remarkable things. The studies described here are very speculative, and definitive answers await further work.

\footnotetext{
1. Walsh, D., Carswell, R.F. \& Weymann, R.R. Nature 279, 381 (1979).

2. Weymann, R.T. et al. Nature 285, 643 (1980)

3. Weedman, D.W., Green, R.F. \& Weymann, R.J. Nature (submitted)

4. Young, P.J., Gunn, J.E., Kristian, J., Oke, J.B. \& Westphall, J.A. Astrophys J. 241, 507 (1980)

5. Tyson, J.A. Astrophys. J. Lett. 248, L89 (1981).

6. Avni, Y Astrophys.

7. Paczynski, B. \& Górski, K. Astrophys, J. Lett. 248, L10 (1981)
}

8. Burbidge, E.M. et al. Astrophys. J. Lett. 242, L55 (1980) 